\title{
The Occurrence of Primary Hepatic Adenoma in Deceased Donor Renal Transplant Recipient
}

\author{
Yu-Tso Liao, Yung-Ming Jeng, Yu-Hsin Lee, Meng-Kun Tsai, Ruy-Heng Hu, Ming-Chih Ho
}

Division of General Surgery; Department of Surgery (YTL, YHL, MKT, RHH, MCH) and Department of Pathology (YMJ), National Taiwan University Hospital, Yuan-Lin Branch, Yuan-Lin, Taiwan, Republic of China

\section{ABSTRACT}

Main findings: We reported a case of new-onset, multi-focal hepatic adenoma in an 18 year-old man with no classic risk factors occurring forty months after a renal transplant from a cadaver donor. Histopathology of the adenoma was examined and genotype and phenotype were also analyzed. Histopathologic examination of the adenoma showed no malignancy. Genotype and phenotype analysis revealed no HNF1 $\alpha$ or $\beta$-catenin gene mutations and no inflammatory infiltration. The patient was well and disease-free postoperatively.

Case hypothesis: Hepatic adenoma occurs mostly in those taking oral contraceptives or androgenic-anabolic steroids or in those with hereditary diseases. Hepatic adenoma in a renal transplant recipient is rare and has only been reported in one case with glycogen storage disease type Ia. Immunosuppressive treatment might have contributed to the development of the neoplasm.

Promising future implications: Although malignant change occurs most often in $\beta$-catenin gene mutation hepatic adenoma, surgical resection of the adenoma in a patient under immunosuppressive therapy should be considered in order to avoid the possibility of malignant transformation or hemorrhagic rupture.

\section{ARTICLE INFO}

\author{
Key words: \\ Adenoma; Liver Cell; Kidney \\ Transplantation; Immunosup- \\ pressive Agents
}

Int Braz J Urol. 2014; 40: 118-22

Submitted for publication:

June 06, 2013

Accepted after revision:

July 17, 2013

\section{INTRODUCTION}

The occurrence of hepatic adenoma is rare. Only a single case of hepatic adenoma in a renal transplant recipient has previously been reported (1), and the patient involved suffered from glycogen storage disease type Ia, a previously reported risk factor. We here describe a case of new onset, multi-focal hepatic adenoma in a renal transplant recipient who had no classical risk factors.

\section{Case hypothesis and rational}

A man suffered from an idiopathic, afebrile, rapid progression of renal insufficiency with initial presentation of oliguria at the age of 18. After three years of hemodialysis, the patient underwent a successful renal transplant from a cadaver donor. Immunosuppressant therapy consisted of prednisolone (Predonin ${ }^{\circledR}$ ), mycophenolic acid (Cellcept ${ }^{\circledast}$ ), sirolimus (Rapammune ${ }^{\circledR}$ ) and tacrolimus (Prograft ${ }^{\circledR}$ ). No induction therapy was used. For the transplant follow-up, the patient underwent monthly laboratory studies including whole blood and renal function tests. Post-transplant creatinine levels were within normal limits. Forty months after transplantation, two hepatic tumors not seen in pre-operative imaging studies were found incidentally during regular ultrasonography: 
a $5 \mathrm{~cm}$ hetero-echoic mass at the left lateral segment and a $2 \mathrm{~cm}$ hypo-echoic mass in the right lobe. MRI showed one $23.4 \mathrm{~mm}$ nodule at Segment 3 with hypointensity on T1W and hyperintensity on T2W image. Two nodules at Segments 6 and 7 showed isointensity on the T1W and hyperintensity on the T2W image. Serum alpha-fetoprotein was $2.50 \mathrm{ng} / \mathrm{mL}$, that is, within normal limits. The patient was otherwise healthy and had no known predisposing hereditary disease such as glycogen storage disease, and did not take any medications prior to the renal transplant. Viral hepatitis tests were negative for hepatitis B and C. Ultrasonography-guided biopsy showed a low-grade dysplastic nodule. Due to suspected malignancy, the patient underwent segmentectomy of S6 and S7 and partial segmentectomy of S3. Grossly, the tumor was yellow in color with focal hemorrhage. No capsule was seen. Microscopically the tumor had blank-looking nuclei and was arranged in trabeculae. The non-tumorous part was unremarkable. The pathologic diagnosis was hepatic ade- noma without evidence of malignancy. Genotype analysis showed no mutations in either HNF1 $\alpha$ or $\beta$-catenin. Histopathology showed no evidence of inflammatory infiltrate (Figure-1). At the present time, thirty six months after surgery, the patient is well and disease-free.

The IL- 6 pathway plays an important role in regulation of the inflammatory status of hepatic adenoma. The deletion of 560-571 of exon- 6 of the IL6ST gene that encodes the signaling co-receptor gp130 of IL-6 may lead to a persistent inflammation in the absence of IL-6 (2). Based on this concept, the somatic DNA sequences in exon-6 of the IL6ST gene were identified in our study. Genomic DNA in tumor tissue was purified by QIAamp DNA Mini Kit (Qiagen). The genomic DNA was amplified IL6ST exon 6 by touchdown polymerase chain reaction with DNA templates from the patient. The purified PCR products were directly sequenced using Applied Biosystems 3730 DNA Analyzer. DNA sequencing was performed using primers provided in Table-1. The sequences

Figure 1 - Immunohistochemistry analyses (200X). (A) Hematoxylin and eosin (H\&E) stain; (B) L-FABP immunostaining. Cytoplasmic and nuclear L-FABP expression was present in the tumor; (C) $\beta$-catenin immunostaining. $\beta$-catenin-inactivated normal membranous staining of hepatocytes was similar in the tumor and non-tumor liver; (D) SAA immunostaining. SAA staining was absent in the liver specimen. These results indicate that the patient's HA belonged to the no mutation, no inflammation HA subtype.
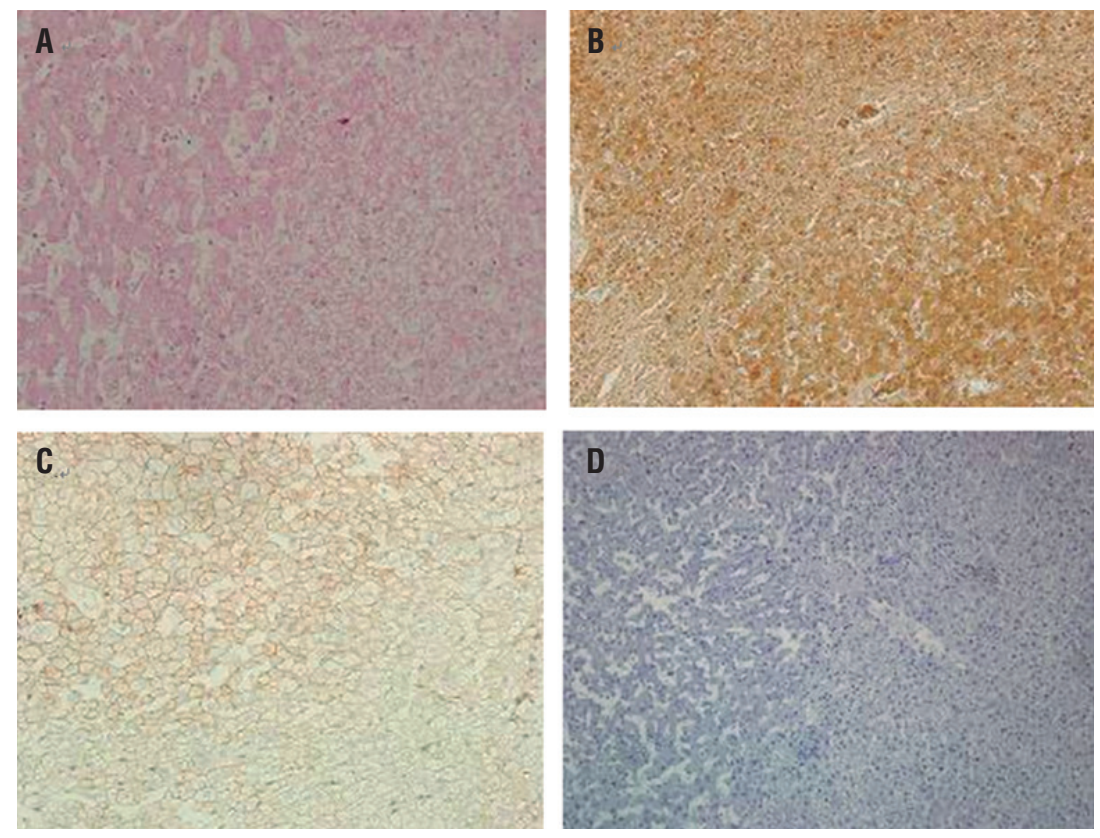
Table 1 - Sequence primer $5^{\prime}->3^{\prime}$.

IL6ST EXON 6 genomic DNA amplification and sequencing

TAGTGACCAGCAGTTATATTGCAA

CGACTACAGTGTCAAATAAACTCTCA

of HA of our case represented the IL6ST-wide-type sequence without any mutation (Figure-2).

Discussion and future perspectives

Hepatic adenoma (HA) is a rare, benign neoplasm with an estimated incidence of $<5$ cases per million persons, but a higher incidence of 1-3 per 100,000 young women with a history of oral contraceptive use (1-3). Patients diagnosed with HA are typically younger women with long-standing oral contraceptive use. Other possible etiologies for HA include androgenic-anabolic steroid use, congenital diseases including glycogen storage disease type I, III and IV, Klinefelter's syndrome, and familial adenomatous polyposis, and some drugs including clomiphene or danazol. The potential for malignant change is extremely low. Only a single case of hepatic adenoma in a renal transplant recipient who suffered from glycogen storage disease type Ia has been previously reported (4). The current case, to our knowledge, is the first report of primary HA developing in a cadaveric donor renal transplant male recipient with a previous healthy liver and no known risk factors.

In our case, no hepatic lesions were visible in the pre-transplant abdominal CT. In the follow-up period, immunosuppressants including prednisolone, mycophenolic acid, sirolimus, and tacrolimus were administered, and the patient tolerated these drugs well. No previous report has demonstrated any correlation between HA and the specific immunosuppressants taken by our patient. In renal transplant recipients, adenomas occur rarely, and the vast majority appears in nephrogenic systems (5) and are related to the use of azathioprine (6). Three cases of post-transplant HA have been reported (7-9) that were attributed to the use of cyclosporine (7). Immunosuppressive effects increase the incidence of malignancy in transplant patients (10). The effect of immunosuppressants on benign adenomas development has not been well studied.

The typical characteristic of HA on ultrasonography is a well-demarcated hyper echoic lesion that tends to heterogenous echogenicity if central necrosis or hemorrhage is present. MRI has advantages in distinguishing between different types of hepatic neoplasms; however the findings for HA vary, and some difficulties exist in distinguishing between HA, focal nodular hyperplasia and well-differentiated hepatocellular carcinoma (11). And the efficacy of percutaneous liver biopsy is limited

Figure 2 - The somatic DNA sequences in exon-6 of the IL6ST gene of our case represented the wide type sequence without any mutation.

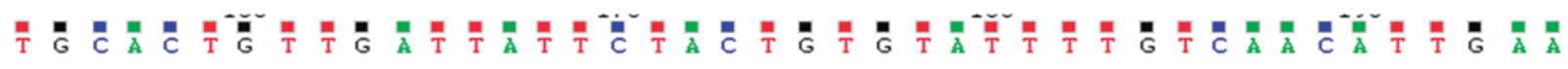

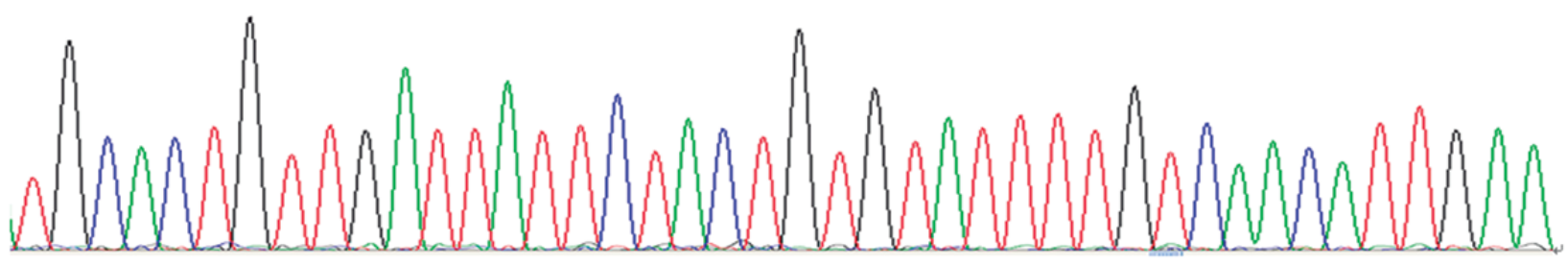


with a preoperative accuracy about 50\% $(11,12)$. Definite diagnosis relies on surgical resection and histopathology.

The incidence of hemorrhage increases in proportion to the tumor size, especially when the size is above $3-4 \mathrm{~cm}^{3}$. Usually rupture of the HA presents with acute abdominal pain and instability of vital signs, and this presentation might be misinterpreted in an immunocompromised patient (13). It has been suggested that the higher incidence of malignancy seen in transplant patients is due to long-term immunosuppressive therapy (14). Therefore surgical resection for a hepatic tumor of undetermined nature in an immunocompromised patient such as our transplant patient is a reasonable option.

HA can be classified into four groups: Group 1, hepatocyte nuclear factor 1 alpha mutations (HNF1 $\alpha$ ); Group 2, $\beta$-catenine mutations; Group 3, presence of inflammatory infiltrate; Group 4 , absence of inflammatory infiltrate (15). Of these groups, $\beta$-catenine-activated HA has the highest correlation with hepatocellular carcinoma.

The absence of liver-type fatty acid binding protein (L-FABP) indicates HNF1 $\alpha$ mutations and can be used to classify Group 1. A combination of GLUL overexpression and nuclear $\beta$-catenin staining indicates $\beta$-catenin-activating mutations and can be used to classify Group 2. Positive serum amyloid A protein (SAA2) staining can be used to classify Group 3, the inflammatory filtrate group (16). In our patient, HNF $1 \alpha, \beta$-catenin, and SAA immunostaining were all absent in the liver specimen and the immunostaining analysis showed both cytoplasmic and nuclear expression of L-FABP. Therefore, these results showed that our case belongs to Group 4 (no mutations, no inflammatory infiltrate), a group that represents only $5-10 \%$ of all HA cases. The clinical relationship between Group 4 HA and immunosuppressants use is unknown at present and should be studied further.

In the current case, we have reported the occurrence of new onset, multi-focal HA forty months following a renal transplant from a cadaver donor in a young man free from classic risk factors. The lack of previously reported risk factors for development of the adenoma leaves the suspicion that the immunosuppressive treatment given to the patient contributed to its development. Surgical resection of the adenoma seemed advisable because of the possibility of hemorrhagic rupture and, in an immunosuppressed patient, of malignant transformation.

\section{CONFLICT OF INTEREST}

None declared.

\section{REFERENCES}

1. Deneve JL, Pawlik TM, Cunningham S, Clary B, Reddy S, Scoggins CR, et al.: Liver cell adenoma: a multicenter analysis of risk factors for rupture and malignancy. Ann Surg Oncol. 2009; 16: 640-8.

2. Rebouissou S, Amessou M, Couchy G, Poussin K, Imbeaud $\mathrm{S}$, Pilati $\mathrm{C}$, et al.: Frequent in-frame somatic deletions activate gp130 in inflammatory hepatocellular tumours. Nature. 2009; 457: 200-4.

3. Rooks JB, Ory HW, Ishak KG, Strauss LT, Greenspan JR, Hill AP, et al.: Epidemiology of hepatocellular adenoma. The role of oral contraceptive use. JAMA. 1979; 242: 644-8.

4. Gossmann J, Scheuermann EH, Frilling A, Geiger H, Dietrich CF: Multiple adenomas and hepatocellular carcinoma in a renal transplant patient with glycogen storage disease type $1 \mathrm{a}$ (vonGierke disease). Transplantation. 2001; 72: 343-4.

5. Banyai-Falger S, Maier U, Susani M, Wiener H, Watschinger B, Hörl WH, Banyai M: High incidence of nephrogenic adenoma of the bladder after renal transplantation. Transplantation. 1998; 65: 511-4.

6. Fournier G, Menut P, Moal MC, Hardy E, Volant A, Mangin $P$ : Nephrogenic adenoma of the bladder in renal transplant recipients: a report of 9 cases with assessment of deoxyribonucleicacid ploidy and long-term followup. J Urol. 1996; 156: 41-4.

7. Yarak S, Floriano M, Decico JE, Konishi CT, Michalany NS, Enokihara MY, et al.: First report of a fibro-adenoma in axillary mammary tissue of a renal transplant patient taking cyclosporin. Int J Dermatol. 2007; 46: 407-9.

8. Sakaguchi T, Nakamura S, Suzuki S, Konno H, Fujita K, Suzuki K, et al.: Intracystic hemorrhage of pancreatic serous cystadenoma after renal transplantation: report of a case. Surg Today. 2000; 30: 667-9.

9. Belldegrun A, Servadio C, Nissenkorn I, Liberman UA: Late appearance of parathyroid adenoma in a renal transplant recipient with multiple primary malignancies. J Urol. 1982; 127: 533-4.

10. Buell JF, Papaconstantinou HT, Skalow B, Hanaway MJ, Alloway RR, Woodle ES: De novo colorectal cancer: five-year survival is markedly lower in transplant recipients compared with the generalpopulation. Transplant Proc. 2005; 37: 960-1. 
11. Charny CK, Jarnagin WR, Schwartz LH, Frommeyer HS, DeMatteo RP, Fong $\mathrm{Y}$, et al.: Management of 155 patients with benign liver tumours. Br J Surg. 2001; 88: 808-13.

12. van der Windt DJ, Kok NF, Hussain SM, Zondervan PE, Alwayn IP, de Man RA, et al.: Case-orientated approach to the management of hepatocellular adenoma. Br J Surg. 2006; 93: 1495-502.

13. Benjamin ER, Jim J, Kim TJ, Meals C, Gritsch HA, Tillou A, et al.: Acute care surgery after renal transplantation. Am Surg. 2009; 75: 882-6.

14. Birkeland SA, Storm HH, Lamm LU, Barlow L, Blohmé I, Forsberg B, et al.: Cancer risk after renal transplantation in the Nordic countries, 1964-1986. Int J Cancer. 1995; 60: 183-9.

15. Zucman-Rossi J, Jeannot E, Nhieu JT, Scoazec JY, Guettier C, Rebouissou S, et al.: Genotype-phenotype correlation in hepatocellular adenoma: new classification and relationship with HCC. Hepatology. 2006; 43: 515-24.
16. Bioulac-Sage P, Rebouissou S, Thomas C, Blanc JF, Saric J, Sa Cunha A, et al.: Hepatocellular adenoma subtype classification using molecular markers and immunohistochemistry. Hepatology. 2007; 46: 740-8.

Correspondence address: Ming-Chih Ho, MD, PhD Department of Surgery National Taiwan University Hospital,

No. 7, Chung-Shan South Road Taipei, Taiwan, ROC Fax: + 8862 2381-9723

E-mail: mcho1215@ntu.edu.tw 